\title{
TITLE:
}

\section{On newforms for Kohnen plus spaces}

$\operatorname{AUTHOR}(\mathrm{S})$ :

Ueda, Masaru; Yamana, Shunsuke

\section{CITATION:}

Ueda, Masaru ...[et al]. On newforms for Kohnen plus spaces.

Mathematische Zeitschrift 2008, 264(1): 1-13

\section{ISSUE DATE:}

2008-11

URL:

http://hdl.handle.net/2433/131749

\section{RIGHT:}

The original publication is available at www.springerlink.com; この論文 は出版社版でありません。引用の際には出版社版をご確認ご利用くだ さい。; This is not the published version. Please cite only the published version. 


\title{
ON NEWFORMS FOR KOHNEN PLUS SPACES
}

\author{
MASARU UEDA AND SHUNSUKE YAMANA
}

\begin{abstract}
In this article, we investigate the plus space of level $N$, where $4^{-1} N$ is a square-free (not necessarily odd) integer. This is a generalization of Kohnen's work. We define a Hecke isomorphism $\wp_{k}$ of $M_{k+1 / 2}(4 M)$ onto $M_{k+1 / 2}^{+}(8 M)$ for any odd positive integer $M$. The methods of the proof of the newform theory are this isomorphism, Waldspurger's theorem, and the dimension identity.
\end{abstract}

\section{Introduction}

The purpose of this paper is to establish the theory of newforms for the Kohnen plus space with respect to $\Gamma_{0}(N)$, where $4^{-1} N$ is a squarefree integer. This is a continuation of Kohnen's work (cf. [1]) in the case when $4^{-1} N$ is odd square-free.

Let us describe our results. The space of cusp forms of weight $k+1 / 2$ with respect to $\Gamma_{0}(N)$ is denoted by $S_{k+1 / 2}(N)$ and the Kohnen plus space $S_{k+1 / 2}^{+}(N)$ is defined by

$$
S_{k+1 / 2}^{+}(N)=\left\{g \in S_{k+1 / 2}(N) \mid g(\tau)=\sum_{n \in \mathbb{N},(-1)^{k} n \equiv 0,1} a_{n}(g) q^{n}\right\}
$$

where $q=e^{2 \pi \sqrt{-1} \tau}$. The $\mathbb{C}$-linear map $\wp_{k}$ on formal power series defined by

$$
\sum_{n \in \mathbb{N} \cup\{0\}} a_{n} q^{n} \mid \wp_{k}=\sum_{n \in \mathbb{N} \cup\{0\},(-1)^{k} n \equiv 0,1} a_{n} q^{n} .
$$

gives a Hecke equivalent isomorphism of $S_{k+1 / 2}(N)$ onto $S_{k+1 / 2}^{+}(2 N)$ (see Proposition 3.3) if $N$ is exactly divisible by 4 . We define an operator $U(d)$ by

$$
\sum_{n} a_{n} q^{n} \mid U(d)=\sum_{n} a_{d n} q^{n}
$$

for each positive integer $d$, and put $U_{k}(d)=U(d) \wp_{k}$.

S. Yamana thanks Prof. Ikeda for useful discussion, and he is supported by JSPS Research Fellowships for Young Scientists. 
We define the space of newforms $S_{k+1 / 2}^{\text {new },+}(N)$ for $S_{k+1 / 2}^{+}(N)$ to be the orthogonal complement of

$$
\sum_{p \mid 4^{-1} N}\left(S_{k+1 / 2}^{+}\left(p^{-1} N\right)+S_{k+1 / 2}^{+}\left(p^{-1} N\right) \mid U_{k}\left(p^{2}\right)\right),
$$

where $p$ extends over all prime divisors of $4^{-1} N$, in $S_{k+1 / 2}^{+}(N)$ with respect to the Petersson inner product. Let $\tilde{T}\left(p^{2}\right)$ (resp. $T(p)$ ) be the usual Hecke operator on the space of modular forms of half-integral (resp. integral) weight. The space of newforms for $S_{2 k}\left(\Gamma_{0}\left(4^{-1} N\right)\right)$ is denoted by $S_{2 k}^{\text {new }}\left(4^{-1} N\right)$.

We shall show the following:

Theorem. Suppose that $k$ is positive and $4^{-1} N$ is square-free.

(1) $S_{k+1 / 2}^{+}(N)=\oplus_{a, d \geq 1, a d \mid 4^{-1} N} S_{k+1 / 2}^{\text {new },+}(4 a) \mid U_{k}\left(d^{2}\right)$.

(2) The operators $\tilde{T}\left(p^{2}\right)$ and $U_{k}\left(q^{2}\right)$, where $\left(p, 4^{-1} N\right)=1$ and $q \mid 4^{-1} N$, fix $S_{k+1 / 2}^{\text {new, }}(N)$. Moreover, $S_{k+1 / 2}^{\text {new },+}(N)$ has an orthogonal $\mathbb{C}$-basis which consists of common Hecke eigenforms of these operators.

(3) There is a bijective correspondence, up to scalar multiple, between Hecke eigenforms in $S_{2 k}^{\text {new }}\left(4^{-1} N\right)$ and those in $S_{k+1 / 2}^{\text {new },+}(N)$ in the following way. If $\phi \in S_{2 k}^{\text {new }}\left(4^{-1} N\right)$ is a primitive form, i.e.,

$$
\phi\left|T(p)=\omega_{p} \phi, \quad \phi\right| U(q)=\omega_{q} \phi
$$

for every prime number $p \nmid 4^{-1} N$ and prime divisor $q$ of $4^{-1} N$, then there is a non-zero Hecke eigenform $g \in S_{k+1 / 2}^{\text {new },+}(N)$ such that

$$
g\left|\tilde{T}\left(p^{2}\right)=\omega_{p} g, \quad g\right| U_{k}\left(q^{2}\right)=\omega_{q} g
$$

for every prime number $p \nmid 4^{-1} N$ and prime divisor $q$ of $4^{-1} N$.

Remark. We can also establish the newform theory for a quadratic non-trivial character $\chi$. See Remark 5.1 in $\S 5$.

Let us explain the contents of each section. In $\S 1$ we introduce the notion of modular forms of half-integral weight. Section 2 is an introduction to the theory of the Kohnen plus space. In $\S 3$ we describe the link between the full space of modular forms of half-integral weight and the Kohnen plus space. In $\S 4$ we describe Waldspurger's result, which we use in the proof of main results in $\S 5$. 


\section{Preliminaries}

If $z \in \mathbb{C}$ and $\ell \in \mathbb{Z}$, then let $z^{1 / 2}$ be the square root of $z$ such that $-\pi / 2<\arg z^{1 / 2} \leq \pi / 2$, and put $z^{\ell / 2}=\left(z^{1 / 2}\right)^{\ell}$. Fix an integer $k$. The set $\mathfrak{G}$ consists of all pairs $(\gamma, \phi(\tau))$, where $\gamma=\left(\begin{array}{ll}a & b \\ c & d\end{array}\right)$ is an element of the connected component $\mathrm{GL}_{2}^{+}(\mathbb{R})$ of $\mathrm{GL}_{2}(\mathbb{R})$ and $\phi$ is a holomorphic function on the upper half-plane $\mathfrak{H}$ satisfying

$$
|\phi(\tau)|=(\operatorname{det} \gamma)^{-k / 2-1 / 4}|c \tau+d|^{k+1 / 2}
$$

We define the group law of $\mathfrak{G}$ by

$$
\left(\gamma_{1}, \phi_{1}(\tau)\right) \cdot\left(\gamma_{2}, \phi_{2}(\tau)\right)=\left(\gamma_{1} \gamma_{2}, \phi_{1}\left(\gamma_{2} \tau\right) \phi_{2}(\tau)\right)
$$

For a function $h$ on $\mathfrak{H}$ and $\alpha=(\gamma, \phi(\tau)) \in \mathfrak{G}$, we put $h \mid \alpha(\tau)=$ $\phi(\tau)^{-1} h(\gamma \tau)$.

There exists an injective homomorphism $\Gamma_{0}(4) \rightarrow \mathfrak{G}$ given by

$$
\gamma \mapsto \gamma^{*}=\left(\gamma, j(\gamma, \tau)^{2 k+1}\right), \quad j(\gamma, \tau)=\left(\frac{c}{d}\right) \epsilon_{d}^{-1}(c \tau+d)^{1 / 2}
$$

for $\gamma=\left(\begin{array}{cc}a & b \\ c & d\end{array}\right) \in \Gamma_{0}(4)$. Here $\left(\frac{c}{d}\right)$ is the Kronecker symbol (see [2]) and

$$
\epsilon_{d}=\left\{\begin{array}{lll}
1 & \text { if } d \equiv 1 & (\bmod 4) \\
\sqrt{-1} & \text { if } d \equiv 3 & (\bmod 4)
\end{array}\right.
$$

Fix an integer $N$ and an even Dirichlet character $\chi \bmod N$ such that $\chi^{2}=1$. Put $\chi(\gamma)=\chi(d)$ for $\gamma=\left(\begin{array}{ll}a & b \\ c & d\end{array}\right) \in \Gamma_{0}(N)$. We write $N=2^{e} M$, where $M$ is an odd integer. The 2-primary component of $\chi$ is denoted by $\chi_{2}$. Throughout this paper (except for $\S 4$ ) we suppose the following conditions:

(I) $e$ equals either 2 or 3 ;

(II) the conductor of $\chi_{2}$ equals either 1 or 4 .

Remark 1.1. We can define the Kohnen plus space $M_{k+1 / 2}^{+}(N, \chi)$ without the condition (II). However, $M_{k+1 / 2}^{+}(N, \chi)=\{0\}$ unless the conductor of $\chi_{2}$ equals either 1 or 4 .

We call a holomorphic function $h$ on $\mathfrak{H}$ a modular (resp. cusp) form of weight $k+1 / 2$ with respect to $\Gamma_{0}(N)$ and $\chi$ if $h \mid \gamma^{*}=\chi(\gamma) h$ for every $\gamma \in \Gamma_{0}(N)$ and it is holomorphic (resp. vanishes) at all cusps. The space of modular (resp. cusp) forms of weight $k+1 / 2$ with respect to $\Gamma_{0}(N)$ and $\chi$ is denoted by $M_{k+1 / 2}(N, \chi)\left(\operatorname{resp} . S_{k+1 / 2}(N, \chi)\right)$.

We denote the $n$-th Fourier coefficient of $h$ by $a_{n}(h)$. Put

$$
\mathfrak{D}_{k, \chi}=\left\{m \in \mathbb{N} \cup\{0\} \mid \chi_{2}(-1)(-1)^{k} m \equiv 0,1 \quad(\bmod 4)\right\} .
$$


Definition 1.2. The space $M_{k+1 / 2}^{+}(N, \chi)$ consists of all functions $g \in$ $M_{k+1 / 2}(N, \chi)$ such that $a_{n}(g)=0$ for all $n \notin \mathfrak{D}_{k, \chi}$. Put $S_{k+1 / 2}^{+}(N, \chi)=$ $M_{k+1 / 2}^{+}(N, \chi) \cap S_{k+1 / 2}(N, \chi)$.

When $\chi$ is the trivial character, we write

$$
\begin{array}{ll}
M_{k+1 / 2}(N)=M_{k+1 / 2}(N, \chi), & S_{k+1 / 2}(N)=S_{k+1 / 2}(N, \chi), \\
M_{k+1 / 2}^{+}(N)=M_{k+1 / 2}^{+}(N, \chi), & S_{k+1 / 2}^{+}(N)=S_{k+1 / 2}^{+}(N, \chi) .
\end{array}
$$

For an element $\alpha=\left(\begin{array}{ll}a & b \\ c & d\end{array}\right) \in \mathrm{GL}_{2}^{+}(\mathbb{R})$ and a function $h$ on $\mathfrak{H}$, we put

$$
h \|_{k+1 / 2} \alpha(\tau)=(\operatorname{det} \alpha)^{k / 2+1 / 4}(c \tau+d)^{-k-1 / 2} h(\alpha \tau) .
$$

We define $\tilde{\delta}_{d} \in \mathfrak{G}$ and an operator $U(d)$ on formal power series by

$$
\begin{gathered}
\tilde{\delta}_{d}=\left(\left(\begin{array}{ll}
d_{1} & \\
& 1
\end{array}\right), d^{-k / 2-1 / 4}\right), \\
\sum_{n \in \mathbb{N} \cup\{0\}} a_{n} q^{n} \mid U(d)=\sum_{n \in \mathbb{N} \cup\{0\}} a_{d n} q^{n}
\end{gathered}
$$

for a positive integer $d$. For each positive divisor $Q$ of $M$ such that $Q$ and $M / Q$ are coprime, we choose an element $\gamma_{Q} \in \mathrm{SL}_{2}(\mathbb{Z})$ such that

$$
\gamma_{Q} \equiv \begin{cases}\left(\begin{array}{cc}
0 & -1 \\
1 & 0
\end{array}\right) & \left(\bmod Q^{2}\right) \\
\mathbf{1}_{2} & \left(\bmod \left(Q^{-1} N\right)^{2}\right)\end{cases}
$$

We define operators $\tilde{W}(Q)$ and $\tilde{Y}(Q)$ on $M_{k+1 / 2}(N)$ by

$$
\begin{aligned}
\tilde{W}(Q) & =\gamma_{Q}^{*} \tilde{\delta}_{Q}, \\
\tilde{Y}(Q) & =Q^{-k / 2+3 / 4} U(Q) \tilde{W}(Q) .
\end{aligned}
$$

Put

$$
\begin{gathered}
\tau(N)=\left(\left(\begin{array}{cc}
0 & -1 \\
N & 0
\end{array}\right),\left(N^{1 / 4}(-\sqrt{-1} \tau)^{1 / 2}\right)^{2 k+1}\right) \in \mathfrak{G}, \\
\tilde{Y}\left(2^{e}\right)=2^{-(k / 2-3 / 4) e} U\left(2^{e}\right) \tilde{W}(M) \tau(N) .
\end{gathered}
$$

Note that $\tau(N)^{2}=1$ and

$$
\tilde{W}(Q)^{2}=\epsilon_{Q}^{-2 k-1} \chi_{Q}(-1) \chi_{N / Q}(Q)
$$

on $M_{k+1 / 2}(N)$, where $\chi_{Q}$ and $\chi_{N / Q}$ are the $Q$ and $N / Q$-primary components of $\chi$ respectively (cf. [5, Proposition 1.18]).

The Petersson inner product $\langle g, h\rangle$ is defined by

$$
\langle g, h\rangle=\left[\mathrm{SL}_{2}(\mathbb{Z}): \Gamma_{0}(N)\right]^{-1} \int_{\Gamma_{0}(N) \backslash \mathfrak{H}} g(\tau) \overline{h(\tau)} y^{k-3 / 2} d x d y,
$$

where $g \in S_{k+1 / 2}(N, \chi), h \in M_{k+1 / 2}(N, \chi)$ and $\tau=x+\sqrt{-1} y$. 
Proposition 1.3. Let $m$ be a positive divisor of $N$. Suppose that a complex valued function $h$ on $\mathfrak{H}$ satisfies the following conditions:

(i) $h(\tau+1)=h(\tau)$ for $\tau \in \mathfrak{H}$;

(ii) $h \mid \tilde{\delta}_{m} \in M_{k+1 / 2}(N, \chi)$.

Then the following assertions hold.

(1) If one of the conditions $m \mathfrak{f}\left(\chi^{\prime}\right) \nmid N$ and $4 m \nmid N$ is satisfied, then $h=0$.

(2) If $N$ is divisible by $m \mathfrak{f}\left(\chi^{\prime}\right)$ and $4 m$, then $h \in M_{k+1 / 2}\left(m^{-1} N, \chi^{\prime}\right)$. Here $\chi^{\prime}=\chi(\underline{m})$ and $\mathfrak{f}\left(\chi^{\prime}\right)$ is the conductor of $\chi^{\prime}$.

Proof. See [3, Lemma 7].

Proposition 1.4. Let $p$ be a prime divisor of $M$ with $\operatorname{ord}_{p} M=1$. Suppose that $\chi_{p}=1$. Then the operator $\epsilon_{p}^{2 k+1} p^{-1 / 2} \tilde{Y}(p)$ is an involution on $M_{k+1 / 2}(N, \chi)$. Let $h \in M_{k+1 / 2}(N, \chi)$ and $\epsilon$ either 1 or -1 . Then the following conditions are equivalent:

(i) $\epsilon_{p}^{2 k+1} p^{-1 / 2} h \mid \tilde{Y}(p)=\epsilon h$;

(ii) $a_{n}(h)=0$ if $\left(\frac{(-1)^{k} n}{p}\right)=-\epsilon$.

Proof. Our assertion is the same as [5, Proposition 1.29] if $e=2$ and $h \in S_{k+1 / 2}(N, \chi)$. We can treat the general case on the same line.

\section{The space $M_{k+1 / 2}^{+}(N, \chi)$}

Throughout this paper, we write

$$
\mu_{k, \chi}=\chi_{2}(-1)(-1)^{[(k+1) / 2]}, \quad \nu_{k, \chi}=\mathbf{e}\left((2 k+1)\left(1-\chi_{2}(-1)\right) / 8\right) .
$$

We abbreviate $\mu=\mu_{k, \chi}$ and $\nu=\nu_{k, \chi}$ if there is no fear of confusion.

For each integer $j$, we put

$$
A_{j}=\left(\begin{array}{cc}
1 & 0 \\
4 M j & 1
\end{array}\right)
$$

and write $\mathcal{A}=A_{1}^{*} \in \mathfrak{G}$. For a function $h$ on $\mathfrak{H}$, we define functions $h \mid P_{4}$ and $h \mid P_{8}$ on $\mathfrak{H}$ by

$$
h\left|P_{4}=\sum_{j=0}^{3} h\right| \xi A_{j}^{*}, \quad h\left|P_{8}=h\right| \xi+h \mid \xi^{-1},
$$

where

$$
\xi=\left(\left(\begin{array}{ll}
4 & 1 \\
0 & 4
\end{array}\right), \mathbf{e}\left((2 k+1)\left(2-\chi_{2}(-1)\right) / 8\right)\right) \in \mathfrak{G}
$$


Note that on formal power series

$$
\sum_{n \in \mathbb{N} \cup\{0\}} a_{n} q^{n} \mid P_{8}=2^{1 / 2} \mu\left(\sum_{n \in \mathfrak{D}_{k, \chi}} a_{n} q^{n}-\sum_{n \notin \mathfrak{D}_{k, \chi}} a_{n} q^{n}\right)
$$

(cf. [1, (2)]. There are misprints in [1]. We should replace $\varepsilon$ by $\chi_{2}(-1)$ ).

Definition 2.1. We define the $\mathbb{C}$-linear map $\wp_{k, \chi}$ on formal power series by

$$
\sum_{n \in \mathbb{N} \cup\{0\}} a_{n} q^{n} \mid \wp_{k, \chi}=\sum_{n \in \mathfrak{D}_{k, \chi}} a_{n} q^{n} .
$$

Put $\wp_{k}=\wp_{k, \chi}$ if $\chi$ is the trivial character.

Proposition 2.2. $\quad$ (1) $P_{2^{e}}$ maps the space $M_{k+1 / 2}(N, \chi)$ into itself.

(2) If $e=2$, then we have $P_{4}=P_{8}+P_{8} \mathcal{A}$ on $M_{k+1 / 2}(N, \chi)$.

(3) If $e=3$, then $P_{8}=2^{1 / 2} \mu$ on $M_{k+1 / 2}^{+}(N, \chi)$, and

$$
P_{8}^{2}=2, \quad \wp_{k, \chi}=2^{-3 / 2} \mu P_{8}+2^{-1}
$$

on the space $M_{k+1 / 2}(N, \chi)$.

Proof. We follow the same line of arguments in [1, pp.36-37]. Put

$$
\Delta_{0}=\Delta_{0}(N, \chi)=\left\{\left(\gamma, \chi(d) j(\gamma, \tau)^{2 k+1}\right) \mid \gamma=\left(\begin{array}{ll}
a & b \\
c & d
\end{array}\right) \in \Gamma_{0}(N)\right\} .
$$

For $\alpha \in \mathfrak{G}$ and $h \in M_{k+1 / 2}(N, \chi)$, we put

$$
h\left|\left[\Delta_{0}(N, \chi) \alpha \Delta_{0}(N, \chi)\right]=\sum_{\beta \in B_{\alpha}} h\right| \beta
$$

if there exists a finite set $B_{\alpha} \subset \mathfrak{G}$ such that

$$
\Delta_{0}(N, \chi) \alpha \Delta_{0}(N, \chi)=\bigsqcup_{\beta \in B_{\alpha}} \Delta_{0}(N, \chi) \beta .
$$

Notice that $\xi^{-1} \Delta_{0}(N, \chi) \xi \cap \Delta_{0}(N, \chi)=\Delta_{0}(16 M, \chi)$ and

$$
\left(\begin{array}{cc}
1-2 M & (M-1) / 2 \\
8 M & 1-2 M
\end{array}\right)^{*} \xi\left(\begin{array}{cc}
1 & 0 \\
-8 M & 1
\end{array}\right)^{*}=\xi^{-1} .
$$

Therefore we have

$$
B_{\xi}= \begin{cases}\left\{\xi, \xi A_{1}^{*}, \xi A_{2}^{*}, \xi A_{3}^{*}\right\} & \text { if } e=2 . \\ \left\{\xi, \xi A_{2}^{*}\right\}=\left\{\xi, \xi^{-1}\right\} & \text { if } e=3 .\end{cases}
$$

It follows that

$$
P_{2^{e}}=\left[\Delta_{0}(N, \chi) \xi \Delta_{0}(N, \chi)\right]
$$

on $M_{k+1 / 2}(N, \chi)$. Thus our assertions are immediate (see (2.1)). 
Proposition 2.3. Suppose that $e=3$. The operator $\tilde{Y}(8)$ then maps $M_{k+1 / 2}(N, \chi)$ into $M_{k+1 / 2}^{+}(N, \chi)$, and $(4 \mu \nu)^{-1} \tilde{Y}(8)$ is an involution on $M_{k+1 / 2}^{+}(N, \chi)$. Moreover, for $\epsilon \in\{ \pm 1\}$ and $g \in M_{k+1 / 2}^{+}(N, \chi)$ the following conditions are equivalent:

(i) $(4 \mu \nu)^{-1} g \mid \tilde{Y}(8)=\epsilon g$;

(ii) $a_{n}(g)=0$ if $\left(\frac{\chi_{2}(-1)(-1)^{k} n}{2}\right)=-\epsilon$.

Here, we put

$$
\left(\frac{a}{2}\right)= \begin{cases}1 & \text { if } a \equiv 1 \quad(\bmod 8) \\ -1 & \text { if } a \equiv 5 \quad(\bmod 8) \\ 0 & \text { if } a \equiv 0,4 \quad(\bmod 8) .\end{cases}
$$

Proof. Recall that we choose $\gamma_{M}=\left(\begin{array}{ll}a & b \\ c & d\end{array}\right)$ in $\S 1$. Let $h \in M_{k+1 / 2}(N, \chi)$. Let $\eta=-1$ if $c<0$ and $d<0$, and let $\eta=1$ otherwise. We have

$$
h \mid \tilde{Y}(8)=\eta \operatorname{sgn}(d)\left(\frac{c}{d}\right) \mathbf{e}\left(\frac{2 k+1}{8}\right) \sum_{j=0}^{7} h \|_{k+1 / 2}\left(\begin{array}{cc}
8(b+j d) & -a-j c \\
64 d & -8 c
\end{array}\right) .
$$

Since $b+j d$ and $8 d$ are coprime if $j$ is an odd integer, we can choose integers $p, q, r, s$ and $t$ such that

$$
\left(\begin{array}{cc}
8(b+j d) & -a-j c \\
64 d & -8 c
\end{array}\right)=\left(\begin{array}{ll}
p & q \\
r & s
\end{array}\right)\left(\begin{array}{ll}
8 & t \\
0 & 8
\end{array}\right) .
$$

Note that $r=8 d$ is divisible by $N$. Observing that

$$
p \equiv s \equiv-t \equiv j \quad(\bmod 8), \quad s \equiv-c \equiv-1 \quad(\bmod M),
$$

we have $\chi(s)=\chi(-s)=\chi_{2}(-j)$ and

$$
\left(\frac{r}{s}\right)=\left(\frac{8 d}{-c-d t}\right)=\eta \operatorname{sgn}(d)\left(\frac{c}{d}\right)\left(\frac{2}{j}\right) .
$$

Let us set

$$
h_{i}(\tau)=\sum_{\chi_{2}(-1)(-1)^{k} n \equiv i} a_{(\bmod 8)}(h) q^{n}
$$

for $i=0, \ldots, 7$. Then, using the assumption (II) in $\S 1$, we have

$$
\begin{aligned}
h \mid \tilde{Y}(8) & =h^{\prime}+\mathbf{e}\left(\frac{2 k+1}{8}\right) \sum_{j \in\{1,3,5,7\}} \chi_{2}(-j) \epsilon_{j}^{-2 k-1}\left(\frac{2}{j}\right) h \|_{k+1 / 2}\left(\begin{array}{cc}
8 & -j \\
0 & 8
\end{array}\right) \\
& =h^{\prime}+4 \mu \nu\left(h_{1}-h_{5}\right),
\end{aligned}
$$

where we abbreviate the sum over even $j \in\{0,2,4,6\}$ by $h^{\prime}$.

Observe that $h^{\prime}(\tau+1 / 2)=h^{\prime}(\tau)$. Applying Proposition 1.3 to

$$
h|\tilde{Y}(8)-h| \tilde{Y}(8) \wp_{k} \in M_{k+1 / 2}(N, \chi),
$$


we have $h^{\prime}(\tau+1 / 4)=h^{\prime}(\tau)$. Thus $\tilde{Y}(8) \operatorname{maps} M_{k+1 / 2}(N, \chi)$ to $M_{k+1 / 2}^{+}(N, \chi)$.

Replacing $h$ with $g$ and repeating the same process, we have

$$
g \mid \tilde{Y}(8)^{2}=g^{\prime \prime}+(4 \mu \nu)^{2}\left(g_{1}+g_{5}\right), \quad g^{\prime \prime}\left(\tau+\frac{1}{4}\right)=g^{\prime \prime} .
$$

The second assertion is an application of Proposition 1.3 to the function $g \mid \tilde{Y}(8)^{2}-(4 \mu \nu)^{2} g$. We easily see that (ii) holds if (i) holds. If (ii) holds, then (i) is an application of Proposition 1.3 to $(4 \mu \nu)^{-1} g \mid \tilde{Y}(8)-\epsilon g$.

Lemma 2.4. If $e=3$, then we have

$$
P_{8} \mathcal{A} P_{8}=2^{1 / 2} \mu \mathcal{A} P_{8} \mathcal{A}
$$

on the space $M_{k+1 / 2}(N, \chi)$.

Proof. Following the same line of computation as in $[1, \S 2]$, we have

$$
h \mid \xi A_{t}^{*} \xi=\mathbf{e}\left((2 k+1)\left(\chi_{2}(-1)-2\right) / 4\right) h \|_{k+1 / 2}\left(\begin{array}{cc}
4(1+t M) & 2+t M \\
16 t M & 4(1+t M)
\end{array}\right)
$$

for $h \in M_{k+1 / 2}(N, \chi)$, and

$$
\left(\begin{array}{cc}
4(1+t M) & 2+t M \\
16 t M & 4(1+t M)
\end{array}\right)=\left(\begin{array}{cc}
1-t M-M^{2} & (1+t M)^{2} / 4 \\
-4 M^{2} & 1+t M+M^{2}
\end{array}\right)\left(\begin{array}{ll}
4 & 1 \\
0 & 4
\end{array}\right) A_{t}
$$

for $t \in\{ \pm 1\}$. Put $r_{t}=1+t M+M^{2}$. It follows from (2.2) that

$$
\begin{aligned}
\mathbf{e}\left(\frac{(2 k+1)\left(2-\chi_{2}(-1)\right)}{8}\right) h \mid P_{8} \mathcal{A} P_{8} & =\sum_{s, t \in\{ \pm 1\}} \chi\left(r_{t}\right) \epsilon_{r_{t}}^{-2 k-1}\left(\frac{-1}{r_{t}}\right) h \mid \mathcal{A} \xi A_{t}^{*} A_{2 s}^{*} \\
& =\alpha h \mid \mathcal{A} P_{8} \mathcal{A},
\end{aligned}
$$

where

$$
\alpha=\sum_{t \in\{ \pm 1\}} \chi_{2}(t) \epsilon_{t}^{-2 k-1}\left(\frac{-1}{t}\right)=\mathbf{e}\left(\chi_{2}(-1)(-1)^{k} / 8\right) 2^{1 / 2}
$$

We have thus completed the proof of Lemma 2.4.

\section{A certain isomorphism}

Proposition 3.1 (Kohnen). We have

$$
P_{4}=\nu^{-1} \tilde{Y}(4)
$$

on the space $M_{k+1 / 2}(4 M, \chi)$. Moreover,

$$
M_{k+1 / 2}^{+}(4 M, \chi)=\left\{h \in M_{k+1 / 2}(4 M, \chi)\left|\left(2^{3 / 2} \mu \nu\right)^{-1} h\right| \tilde{Y}(4)=h\right\}
$$

and the following direct sum decomposition holds.

$$
\begin{gathered}
M_{k+1 / 2}(4 M, \chi)=M_{k+1 / 2}^{+}(4 M, \chi) \oplus M_{k+1 / 2}^{-}(4 M, \chi), \\
M_{k+1 / 2}^{-}(4 M, \chi)=\left\{h \in M_{k+1 / 2}(4 M, \chi)\left|\left(2^{1 / 2} \mu \nu\right)^{-1} h\right| \tilde{Y}(4)=-h\right\} .
\end{gathered}
$$


Proof. As is well-known,

$$
U(4)=4^{k / 2-3 / 4}\left[\Delta_{0}(4 M, \chi) \tilde{\delta}_{4}^{-1} \Delta_{0}(4 M, \chi)\right]
$$

(see the proof of Proposition 2.2 for notation). We have

$$
\begin{aligned}
\tilde{Y}(4) & =\left[\Delta_{0} \tilde{\delta}_{4}^{-1} \Delta_{0}\right] \tilde{W}(M) \tau(4 M) \\
& =\left[\Delta_{0}\left(\left(\begin{array}{cc}
1 & -1 \\
0 & 4
\end{array}\right), 4^{k / 2+1 / 4}\right) \Delta_{0}\right] \tilde{W}(M) \tau(4 M) \\
& =\left[\Delta_{0}\left(\left(\begin{array}{cc}
1 & -1 \\
0 & 4
\end{array}\right), 4^{k / 2+1 / 4}\right) \tilde{W}(M) \tau(4 M) \Delta_{0}\right] \\
& =\left[\Delta_{0}\left(\left(\begin{array}{cc}
4 & 1 \\
0 & 4
\end{array}\right), \mathbf{e}((2 k+1) / 8)\right) \Delta_{0}\right]=\nu P_{4}
\end{aligned}
$$

as in the proof of Proposition 2.3. We know that the similar statement holds for the space $S_{k+1 / 2}(4 M, \chi)$, taking [1, Proposition 1] into account. We can treat $M_{k+1 / 2}(4 M, \chi)$ by the same way.

Remark 3.2. In a similar method, we can show that

$$
\nu^{-1} \tilde{Y}(8)=\left[\Delta_{0}(8 M, \chi) \eta \Delta_{0}(8 M, \chi)\right]
$$

where

$$
\eta=\left(\left(\begin{array}{ll}
8 & 1 \\
0 & 8
\end{array}\right), \mathbf{e}\left((2 k+1)\left(2-\chi_{2}(-1)\right) / 8\right)\right) .
$$

The following proposition is important since at the moment, our knowledge of the space $S_{k+1 / 2}(8 M, \chi)$ is incomplete, although there are a number of results available in the space $S_{k+1 / 2}(4 M, \chi)$. For example, fairly good information on $S_{k+1 / 2}(4 M, \chi)$ was obtained in [6].

Proposition 3.3. The map $\wp_{k, \chi}$ induces a $\mathbb{C}$-linear isomorphism of $M_{k+1 / 2}(4 M, \chi)$ onto $M_{k+1 / 2}^{+}(8 M, \chi)$, which maps $S_{k+1 / 2}(4 M, \chi)$ onto $S_{k+1 / 2}^{+}(8 M, \chi)$.

Proof. Put

$$
\varrho_{k, \chi}=2^{-1}(1+\mathcal{A})\left(3-2^{-1 / 2} \mu P_{4}\right) .
$$

The map $\varrho_{k, \chi}$, which sends $M_{k+1 / 2}^{+}(8 M, \chi)$ into $M_{k+1 / 2}(4 M, \chi)$, turns out to be the inverse of $\wp_{k, \chi}$. Indeed, Propositions 2.2 (2) and 3.1 show that

$\wp_{k, \chi} \varrho_{k, \chi}=\left(2^{-3 / 2} \mu P_{8}+2^{-1}\right) \varrho_{k, \chi}=2^{-1}\left(2^{-3 / 2} \mu P_{4}+1\right)\left(3-2^{-1 / 2} \mu P_{4}\right)=1$ on $M_{k+1 / 2}(4 M, \chi)$. It follows that

$$
\begin{aligned}
\left(3-2^{-1 / 2} \mu P_{4}\right) \wp_{k, \chi} & =\left(3-2^{-1 / 2} \mu\left(P_{8}+P_{8} \mathcal{A}\right)\right)\left(2^{-3 / 2} \mu P_{8}+2^{-1}\right) \\
& =1+2^{-1 / 2} \mu P_{8}-2^{-3 / 2} \mu P_{8} \mathcal{A}-2^{-2} P_{8} \mathcal{A} P_{8} .
\end{aligned}
$$

By virtue of Lemma 2.4, we have

$$
\varrho_{k, \chi} \wp_{k, \chi}=\left(1+2^{-1 / 2} \mu P_{8}-\left(2^{-1 / 2} \mu P_{8}-1\right) \mathcal{A}\left(1+2^{-1 / 2} \mu P_{8}\right)\right) / 2 .
$$


Since $P_{8}=2^{1 / 2} \mu$ on $M_{k+1 / 2}^{+}(8 M, \chi)$, we conclude that $\varrho_{k, \chi} \wp_{k, \chi}=1$.

Corollary 3.4. Let $g \in S_{k+1 / 2}(4 M, \chi)$ and $h \in M_{k+1 / 2}(4 M, \chi)$. Write

$$
\begin{gathered}
g=g_{1}+g_{2}, \quad h=h_{1}+h_{2}, \\
g_{1}, h_{1} \in M_{k+1 / 2}^{+}(4 M, \chi), \quad g_{2}, h_{2} \in M_{k+1 / 2}^{-}(4 M, \chi)
\end{gathered}
$$

(see Proposition 3.1 for the definition of $M_{k+1 / 2}^{-}(4 M, \chi)$ ). Then we have

$$
\left\langle g\left|\wp_{k, \chi}, h\right| \wp_{k, \chi}\right\rangle=\left\langle g_{1}, h_{1}\right\rangle+\frac{\left\langle g_{2}, h_{2}\right\rangle}{4} .
$$

Proof. Observing Propositions 2.2, 3.3 and their proofs, we have

$$
\begin{aligned}
\left\langle g\left|\wp_{k, \chi}, h\right| \wp_{k, \chi}\right\rangle & =\left\langle g, h \mid \wp_{k, \chi}^{2}\right\rangle=\left\langle g, h \mid \wp_{k, \chi}\right\rangle \\
& =\left\langle g, h \mid \wp_{k, \chi}(1+\mathcal{A})\right\rangle / 2=\left\langle g, h \mid\left(2^{-3 / 2} \mu P_{4}+1\right)\right\rangle / 2 .
\end{aligned}
$$

Corollary 3.4 is an easy consequence of Proposition 3.1.

We shall use Corollaries 3.4 and 5.2 in a forthcoming paper.

\section{Results of Waldspurger}

We use the notation in [6] in this section. Let $\phi \in S_{2 k}\left(\Gamma_{0}(d)\right)$ be a primitive form. Put $\lambda_{p}=p^{-k+1 / 2} a_{p}(\phi)$ for each prime number $p$. Choose a complex number $\alpha_{p}$ such that $\alpha_{p}+\alpha_{p}^{-1}=\lambda_{p}$ when $p$ and $d$ are coprime. We denote by $\rho_{v}$ the local component at a place $v$ of the automorphic representation determined by $\phi$. Let $S$ be the set of places such that $\rho_{v}$ is not a irreducible principal series.

We assume the following conditions:

$\left(H_{1}\right)$ If $p \notin S$, then $\rho_{p} \simeq \pi\left(\mu_{p}, \mu_{p}^{-1}\right)$ with $\mu_{p}(-1)=1$;

$\left(H_{2}\right) \rho_{2}$ is not supercuspidal or $d$ is divisible by 16 .

Let $V(N)$ be the orthogonal complement of the space spanned by theta functions in $S_{3 / 2}(N)$. The space $S_{k+1 / 2}(N, \phi)$ consists of all functions $g \in S_{k+1 / 2}(N)$ (resp. $V(N)$ ) such that $g \mid \tilde{T}\left(p^{2}\right)=a_{p}(\phi) g$ for every rational prime $p \nmid N$ if $k>1$ (resp. $k=1$ ).

For each rational prime $p$ and non-negative integer $e$, Waldspurger [6] associated to $\phi$ a non-negative integer $\tilde{n}_{p}$ and a set $U_{p}(e, \phi)$ of functions on $\mathbb{Q}_{p}^{\times}$invariant under $\mathbb{Z}_{p}^{\times 2}$, the supports of which lie within $\mathbb{Z}_{p} \cap \mathbb{Q}_{p}^{\times}$. Put $\tilde{N}(\phi)=\prod_{p} p^{\tilde{n}_{p}}$.

Remark 4.1. If $p$ and $2 d$ are coprime, then $U_{p}(0, \phi)=\left\{c_{p}^{0}\left[\lambda_{p}\right]\right\}$, where we define $c_{p}^{0}\left[\lambda_{p}\right]$ in the following way. We put

$$
l_{e}(X)= \begin{cases}\frac{X^{e+1}-X^{-e-1}}{X-X^{-1}} & \text { if } e \geq 0 \\ 0 & \text { if } e<0\end{cases}
$$


for each integer $e$. For each rational prime $p$ and $a \in \mathbb{Q}_{p}^{\times}$, we put

$$
\lambda_{p, a}=l_{\mathfrak{f}_{p}(a)}-\underline{\psi}_{p}\left((-1)^{k} a\right) p^{-1 / 2} l_{\mathfrak{f}_{p}(a)-1}
$$

and

$$
\mathfrak{f}_{p}(a)=\left[\frac{\operatorname{ord}_{p} a+1-\delta_{p 2}}{2}\right]-1+\underline{\psi}_{p}\left((-1)^{k} a\right)^{2},
$$

where $\delta_{i j}$ be the Kronecker delta, and $\underline{\psi}_{p}(a)=1,-1,0$ accordingly as $\mathbb{Q}_{p}(\sqrt{a})$ is $\mathbb{Q}_{p}$, an unramified quadratic extension of $\mathbb{Q}_{p}$ or a ramified quadratic extension of $\mathbb{Q}_{p}$. Then we put $c_{p}^{0}\left[\lambda_{p}\right](a)=\lambda_{p, a}\left(\alpha_{p}\right)$ for every $a \in \mathbb{Q}_{p}^{\times}$.

Let $\mathbb{N}^{s c}$ be the set of positive square-free integers. For each positive integer $n$, the unique element of the set $\mathbb{N}^{s c} \cap n \mathbb{Q}^{\times 2}$ is denoted by $n^{\text {sc }}$. Let $\underline{A}$ be a function on $\mathbb{N}^{s c}$ and $E$ a positive integer which is divisible by $\tilde{N}(\phi)$. Put $e_{p}=\operatorname{ord}_{p} E$ and define a function $g\left(\underline{c_{E}}, \underline{A}\right)$ on $\mathfrak{H}$ by

$$
g\left(\underline{c_{E}}, \underline{A}\right)(\tau)=\sum_{n \in \mathbb{N}} \underline{A}\left(n^{\mathrm{sc}}\right) n^{k / 2-1 / 4} \prod_{p} c_{p}(n) q^{n}
$$

for $\underline{c}_{E}=\left(c_{p}\right) \in \prod_{p} U_{p}\left(e_{p}, \phi\right)$. We write $\bar{U}(E, \phi, \underline{A})$ for the space spanned by functions $g\left(\underline{c_{E}}, \underline{A}\right)$ for $\underline{c_{E}} \in \prod_{p} U_{p}\left(e_{p}, \phi\right)$.

Waldspurger showed the following results in slightly more general situations.

Theorem 4.2 (cf. [6, Theorem 1]). Suppose that $\phi$ satisfies the hypotheses $\left(H_{1}\right)$ and $\left(H_{2}\right)$. There exists a function $\underline{A^{\phi}}: \mathbb{N}^{s c} \rightarrow \mathbb{C}$ which satisfies the following conditions:

(i) for every $t \in \mathbb{N}^{s c}$

$$
\underline{A^{\phi}}(t)^{2}=L\left(\phi \psi_{(-1)^{k} t}, 1 / 2\right) \varepsilon\left(\psi_{(-1)^{k} t}, 1 / 2\right),
$$

where $L\left(\phi \psi_{(-1)^{k} t}, 1 / 2\right)$ is the central critical value of the $L$ function of $\phi$ twisted with the quadratic character corresponding to $\mathbb{Q}\left(\sqrt{(-1)^{k} t}\right)$

(ii) for every positive integer $N$

$$
S_{k+1 / 2}(N, \phi)=\oplus_{\tilde{N}(\phi)|E| N} \bar{U}\left(E, \phi, \underline{A^{\phi}}\right),
$$

where $E$ extends over all positive divisors of $N$ divisible by $\tilde{N}(\phi)$.

Proposition 4.3. Assume that $d$ is square-free. Put $t_{p}=\log \alpha_{p} / \log p$ or $\log \lambda_{p} / \log p$ accordingly as $p \notin S$ or $p \in S$. Then the following assertions hold.

(1) $S$ consists of the infinite place and all prime divisors of $d$.

(2) If $p \notin S, \rho_{p}$ is the principal series $\pi\left(||_{p}^{-t_{p}},||_{p}^{t_{p}}\right)$. 
TABLE 1

\begin{tabular}{c|c|c|c} 
& $U_{p}(0, \phi)$ & $U_{p}(1, \phi)$ & $U_{p}(2, \phi)$ \\
\hline $2 \neq p \notin S$ & $\left\{c_{p}^{0}\left[\lambda_{p}\right]\right\}$ & $\left\{c_{p}^{\prime}\left[\alpha_{p}\right]\right\}$ & \\
$2 \neq p \in S$ & $\emptyset$ & $\left\{c_{p}^{s}\left[\lambda_{p}\right]\right\}$ & \\
$2=p \notin S, \alpha_{2} \neq \pm 1$ & $\emptyset$ & $\emptyset$ & $\left\{c_{2}^{\prime}\left[\alpha_{2}\right], c_{2}^{\prime}\left[\alpha_{2}^{-1}\right]\right\}$ \\
$2=p \notin S, \alpha_{2} \in\{ \pm 1\}$ & $\emptyset$ & $\emptyset$ & $\left\{c_{2}^{\prime}\left[\alpha_{2}\right], c_{2}^{\prime \prime}\left[\alpha_{2}\right]\right\}$ \\
$2=p \in S$ & $\emptyset$ & $\emptyset$ & $\left\{c_{2}^{s}\left[\lambda_{2}\right]\right\}$
\end{tabular}

(3) If $p$ is a rational prime in $S$, then $\lambda_{p}$ is either $p^{-1 / 2}$ or $-p^{-1 / 2}$, and $\rho_{p}$ is the special representation $\sigma\left(||_{p}^{-t_{p}},||_{p}^{t_{p}}\right)$.

(4) $S_{3 / 2}(4 d)=V(4 d)$.

(5) $\tilde{n}_{p}=\operatorname{ord}_{p} d$ or 2 accordingly as $p$ is odd or $p=2$.

(6) Assume that $N$ is divisible by $d$ and 4. Then all functions $g \in$ $S_{k+1 / 2}(N, \phi)$ satisfies the following conditions for each prime number $p \in S$ :

(a) If $\underline{\psi}_{p}\left((-1)^{k} n\right)=p^{1 / 2} \lambda_{p}$, then $a_{n}(g)=0$;

(b) $g \mid U\left(p^{2}\right)=p^{k-1 / 2} \lambda_{p} g$ if $(2 p)^{-1} N$ and $p$ are coprime.

Remark 4.4. From Proposition 4.3 (2) and (3), the hypotheses $\left(H_{1}\right)$ and $\left(H_{2}\right)$ are automatically satisfied if $d$ is square-free.

Proof. Our assertions (1), (2) and (3) are well-known. See [4, §3 Corollary] for (4). Our assertion (5) directly follows from the definition of $\tilde{n}_{p}$. Our assertion (a) is a special case of [6, Proposition 19].

The set $U_{p}(e, \phi)$ specifies as in Table 1 . For each $\delta \in \mathbb{C}$ and odd rational prime $p$, the definitions of functions $c_{p}^{\prime}[\delta], c_{p}^{s}[\delta], c_{2}^{\prime}[\delta], c_{2}^{\prime \prime}[\delta]$ and $c_{2}^{s}[\delta]$ can be found in [6] (for $c_{p}^{0}[\delta]$ see remark 4.1). Let us note that $c_{p}^{s}\left[\lambda_{p}\right]\left(a p^{2}\right)=\lambda_{p} c_{p}^{s}\left[\lambda_{p}\right](a)$ for every $a \in \mathbb{Z}_{p} \cap \mathbb{Q}_{p}^{\times}$. We thus obtain the assertion (b) in view of Theorem 4.2 and Table 1.

If $d$ is square-free, then we define a function $g^{\phi}$ on $\mathfrak{H}$ by

$$
g^{\phi}(\tau)=\sum_{n \in \mathbb{N}} \underline{A^{\phi}}\left(n^{\mathrm{sc}}\right) n^{k / 2-1 / 4} \prod_{p \in S} c_{p}^{s}\left[\lambda_{p}\right](n) \prod_{p \notin S} \lambda_{p, n}\left(\alpha_{p}\right) q^{n}
$$

(see Remark 4.1 and the proof of Proposition 4.3 for $c_{p}^{s}\left[\lambda_{p}\right]$ and $\lambda_{p, n}$ ).

Proposition 4.5. If $2 M$ is square-free and $d$ is a positive divisor of $2 M$, then

$$
S_{k+1 / 2}(4 M, \phi)=\oplus_{b \mid 2 d^{-1} M} \mathbb{C} g^{\phi} \mid U\left(b^{2}\right),
$$

where $b$ extends over all positive divisors of $2 d^{-1} M$. 
Proof. If $d$ is odd, then we can easily check that

$$
\lambda_{2, a}\left(\alpha_{2}\right)= \begin{cases}\frac{\alpha_{2} c_{2}^{\prime}\left[\alpha_{2}\right](a)-\alpha_{2}^{-1} c_{2}^{\prime}\left[\alpha_{2}^{-1}\right](a)}{\alpha_{2}-\alpha_{2}^{-1}} & \text { if } \alpha_{2} \neq \pm 1 \\ \alpha_{2} c_{2}^{\prime \prime}\left[\alpha_{2}\right](a) & \text { if } \alpha_{2} \in\{ \pm 1\}\end{cases}
$$

for every $a \in \mathbb{Q}_{2}^{\times}$. Remarks $4.1,4.4$ and Theorem 4.2 thus show that $g^{\phi} \in S_{k+1 / 2}(\tilde{N}(\phi), \phi)$. It follows from an easy computation that

$$
c_{p}^{\prime}\left[\alpha_{p}\right](a)=\left(\lambda_{p, p^{2} a}\left(\alpha_{p}\right)-\alpha_{p}^{-1} \lambda_{p, a}\left(\alpha_{p}\right)\right) \times \begin{cases}\alpha_{p}^{-1} & \text { if } p \neq 2 \\ 1 & \text { if } p=2\end{cases}
$$

for every $p \notin S$ and $a \in \mathbb{Q}_{p}^{\times}$. Theorem 4.2, Proposition 4.3 (5) and Table 1 show that $S_{k+1 / 2}(4 M, \phi)$ is spanned by functions $g^{\phi} \mid U\left(b^{2}\right)$ with positive divisors $b$ of $2 d^{-1} M$.

We have only to show that these functions are linearly independent. For a positive integer $N$, the symbol $t(N)$ stands for the number of prime divisors of $N$. We see that

$$
\operatorname{dim}_{\mathbb{C}} S_{k+1 / 2}(4 M, \phi) \leq 2^{t\left(2 d^{-1} M\right)} .
$$

In view of Proposition 4.3 (4), we know that

$$
S_{k+1 / 2}(4 M)=\oplus_{d \mid 2 M, \phi \in \operatorname{Prm}_{2 k}(d)} S_{k+1 / 2}(4 M, \phi),
$$

where $\operatorname{Prm}_{2 k}(d)$ denotes the set of primitive forms in $S_{2 k}\left(\Gamma_{0}(d)\right)$. Since

$$
S_{2 k}\left(\Gamma_{0}(2 M)\right)=\left.\oplus_{b, d \geq 1, b d \mid 2 M, \phi \in \operatorname{Prm}_{2 k}(d)} \mathbb{C} \phi\right|_{2 k}\left(\begin{array}{cc}
b & \\
& 1
\end{array}\right),
$$

it follows that

$$
\operatorname{dim}_{\mathbb{C}} S_{2 k}\left(\Gamma_{0}(2 M)\right)=\sum_{d \mid 2 M} 2^{t\left(2 d^{-1} M\right)} \sharp \operatorname{Prm}_{2 k}(d) .
$$

Since [4, $§ 3$ Corollary] shows that

$$
\operatorname{dim}_{\mathbb{C}} S_{2 k}\left(\Gamma_{0}(2 M)\right)=\operatorname{dim}_{\mathbb{C}} S_{k+1 / 2}(4 M),
$$

(4.2) and (4.3) show that the equality holds in (4.1). The proof of Proposition 4.5 is now complete.

\section{Proof of Main Theorem}

We now prove Theorem (see Introduction for notation and the statement). If $e=3$, then Propositions 3.3, 4.5 and (4.2) show that

$$
\begin{aligned}
S_{k+1 / 2}^{+}(N)=S_{k+1 / 2}\left(2^{-1} N\right) \mid \wp_{k} & =\oplus_{d \mid 4^{-1} N, \phi \in \operatorname{Prm}_{2 k}(d)} S_{k+1 / 2}\left(2^{-1} N, \phi\right) \mid \wp_{k} \\
& =\oplus_{b, d \geq 1, b d \mid 4^{-1} N, \phi \in \operatorname{Prm}_{2 k}(d)} \mathbb{C} g^{\phi} \mid \wp_{k} U_{k}\left(b^{2}\right) .
\end{aligned}
$$


Note that (5.1) also holds when $e=2$. It follows that

$$
S_{k+1 / 2}^{\text {new },+}(N)=\oplus_{\phi \in \operatorname{Prm}_{2 k}\left(4^{-1} N\right)} \mathbb{C} g^{\phi} \mid \wp_{k} .
$$

Proposition 4.3 (b) shows that $g^{\phi}\left|\wp_{k} U_{k}\left(q^{2}\right)=\omega_{q} g^{\phi}\right| \wp_{k}$ for every prime divisor $q$ of $4^{-1} N$. We thus observe that functions $g^{\phi} \mid \wp_{k}$ satisfy the conditions of (3). We are led to our assertion (1) and (2) by virtue of (5.1) and (5.2).

Remark 5.1. We can establish the newform theory for an even quadratic character $\chi$. Letting $\mathfrak{f}$ be the conductor of $\chi$ and observing that $M_{k+1 / 2}^{+}(N) \mid U\left(\mathfrak{f}^{\prime}\right)=M_{k+1 / 2}^{+}(N, \chi)$, where $\mathfrak{f}^{\prime}$ is either $\mathfrak{f}$ or $\mathfrak{f} / 4$ according as $\mathfrak{f}$ is odd or even, we can define the space of newforms for $S_{k+1 / 2}^{+}(N, \chi)$ by

$$
S_{k+1 / 2}^{\text {new },+}(N, \chi)=S_{k+1 / 2}^{\text {new },+}(N) \mid U\left(\mathfrak{f}^{\prime}\right),
$$

and can generalize our result as in [1].

Corollary 5.2. Under the notation as in Theorem, the following assertions holds.

(1) On the space $S_{k+1 / 2}^{\text {new, }}(N)$, we have

$$
p^{1-k} U_{k}\left(p^{2}\right)= \begin{cases}-\epsilon_{p}^{2 k+1} p^{-1 / 2} \tilde{Y}(p) & \text { if } 2 \neq p \mid 4^{-1} N . \\ -(4 \mu \nu)^{-1} \tilde{Y}(8) & \text { if } 2=p \mid 4^{-1} N .\end{cases}
$$

(2) Let $p$ be a prime divisor of $4^{-1} N$. If $g \in S_{k+1 / 2}^{+}\left(p^{-1} N\right)$ is an eigenfunction of the operator $\tilde{Y}\left(p^{\operatorname{ord}_{p} N}\right)$, then $g=0$.

Proof. Combining Propositions 1.4, 2.3, 4.3 (6) with (5.2), we can establish our assertion (1).

If $p$ is odd, then (1.1) and the definition of $\tilde{Y}(p)$ show that $g \mid U(p)$ and $g|\tilde{W}(p)=g| \tilde{\delta_{p}}$ are linearly dependent. This contradicts Theorem (1) for $g \mid \tilde{\delta}_{p} U(p)=p^{k / 2+1 / 4} g$. If $p=2$, then taking Proposition 3.1 into account, we can show that $g \mid U(4) \tilde{\delta}_{2}$ and $g \mid U(8)$ are linearly dependent, which contradicts Theorem (1).

\section{REFERENCES}

[1] W. Kohnen, Newforms of half-integral weight, J. reine angew. Math. 333 (1982) 32-72.

[2] T. Miyake, Modular Forms, Springer, 1989.

[3] J. P. Serre and H. M. Stark, Modular forms of weight $1 / 2$, Springer Lec. notes in Math. 627 (1977) 27-67.

[4] M. Ueda, The decomposition of the spaces of cusp forms of half-integral weight and trace formula of Hecke operators, J. Math. Kyoto Univ. 28, (1988) 505555 . 
[5] M. Ueda, On twisting operators and newforms of half-integral weight, Nagoya. Math. J., 131, (1993) 135-205.

[6] J. L. Waldspurger, Sur les coefficients de Fourier des formes modulaires de poids demi-entier, J. Math. Pures Appl. 60, (1981) 375-484.

Department of Mathematics, Faculty of Science, Nara Women's UNIVERSITY, NARA 630 JAPAN

E-mail address: m-ueda@cc.nara-wu.ac.jp

Graduate school of mathematics, Kyoto University, Kitashirakawa, KYоTO, 606-8502, JAPAN

E-mail address: yamana07@math.kyoto-u.ac.jp 